\title{
A case of peritoneal malignant mesothelioma following radiation therapy for cervical cancer
}

\author{
MITSUTAKE YANO $^{1}$, YUJI IKEDA ${ }^{2}$, TOMOMI KATO $^{1}$, MIKA SAKAKI $^{1}$, \\ SHO SATO $^{2}$, AKIRA YABUNO ${ }^{2}$, EITO KOZAWA ${ }^{3}$ and MASANORI YASUDA ${ }^{1}$ \\ Departments of ${ }^{1}$ Pathology and ${ }^{2}$ Gynecologic Oncology, Saitama Medical University \\ International Medical Center, Hidaka, Saitama 350-1298; ${ }^{3}$ Department of Diagnostic Radiology, \\ Saitama Medical University Hospital, Moroyama, Saitama 350-0495, Japan
}

Received June 16, 2017; Accepted November 24, 2017

DOI: $10.3892 / \mathrm{mco} .2017 .1525$

\begin{abstract}
The present study presents a case of peritoneal malignant mesothelioma (PMM) following radiation therapy for cervical cancer. A 34-year-old Japanese woman, without asbestos exposure, was referred to the Department of Gynecologic Oncology, Saitama Medical University International Medical Center due to a cervical mass, and was diagnosed with cervical squamous cell carcinoma (SCC). The serum levels of tumor markers, including SCC antigen and cancer antigen 125 (CA125) were $229.0 \mathrm{ng} / \mathrm{ml}$ and $54.4 \mathrm{U} / \mathrm{ml}$, respectively. The patient underwent concurrent chemoradiotherapy (CCRT), and a complete response was achieved. After 54 months, ascites was found at the rectouterine pouch, but peritoneal cytology suggested reactive mesothelial cell. After 62 months of CCRT, magnetic resonance imaging revealed masses in both the salpinges. The serum levels of SCC and CA125 were $0.9 \mathrm{ng} / \mathrm{ml}$ and $506.1 \mathrm{U} / \mathrm{ml}$, respectively. Following this, left salpingectomy and peritoneal biopsy were performed laparoscopically. Histologic examination revealed atypical mesothelial cells with no continuity of background tubal epithelium. Immunohistochemistry showed positive staining for calretinin, thrombomodulin, mesothelin and glucose transporter 1. Based on these findings, the patient was diagnosed with PMM epithelioid type and underwent systemic chemotherapy; stable disease status has been obtained for 3 months. This case demonstrates the possibility of PMM
\end{abstract}

Correspondence to: Dr Mitsutake Yano, Department of Pathology, Saitama Medical University International Medical Center, 1397-1 Yamane, Hidaka, Saitama 350-1298, Japan

E-mail: yano1210@oita-u.ac.jp

Abbreviations: PMM, peritoneal malignant mesothelioma; SCC, squamous cell carcinoma; CA125, cancer antigen 125; CCRT, concurrent chemoradiotherapy; MRI, magnetic resonance imaging; FIGO, International Federation of Gynecology and Obstetrics; IHC, immunohistochemistry; BAP1, BRCA1-associated protein-1; FISH in situ hybridization

Key words: peritoneal malignant mesothelioma, cervical cancer, radiation, secondary malignancy, case report occurrence within 10 years after radiotherapy, and indicates the importance of histological and immunohistochemical examination, particularly in cases of an atypical tumorigenesis pattern from the primary cancer.

\section{Introduction}

Peritoneal malignant mesothelioma (PMM) is a rare and aggressive neoplasm that arises from the lining mesothelial cells of the peritoneum and spreads extensively within the confines of the abdominal cavity. Even though asbestos is the most important factor for mesothelioma, recent studies have focused on other causal factors, including radiation (1). The incidence of secondary malignancies after radiation therapy is increasing because of the improved prognosis of cancer survivors owing to the development of anticancer therapies. Radiation plays a pivotal role in treating several cancers in organs such as the uterine cervix, testis, breast, and prostate (2). Therefore, secondary malignancies after radiation are recently recognized as a major problem (3). Secondary cancers, such as bladder, kidney, rectal, uterine corpus, and ovarian cancer, after radiation therapy for uterine cervical cancer, are well-known events $(4,5)$. We herein report our experience with a case of PMM after radiation therapy for cervical cancer, along with a review of the pertinent literature.

\section{Case report}

A 34-year-old Japanese woman, gravida 2, para 2, without any history of cancer or asbestos exposure, had visited another clinic because of atypical genital bleeding for a month; she was referred to our hospital for evaluation of a uterine cervical mass. T2-weighted magnetic resonance imaging (MRI) revealed the presence of a $29 \times 21 \mathrm{~mm}$ mass at the uterine cervix. The tumor was diagnosed as squamous cell carcinoma (SCC), non-keratinizing type, by target biopsy under colposcopy (Fig. 1A). The serum levels of tumor markers of SCC antigen and cancer antigen 125 (CA125) were $229.0 \mathrm{ng} / \mathrm{ml}$ and $54.4 \mathrm{U} / \mathrm{ml}$, respectively. The patient was diagnosed with uterine cervical cancer, at International Federation of Gynecology and Obstetrics (FIGO) stage IIB (T2bN0M0). The patient underwent concurrent chemoradiotherapy (CCRT), with 
A

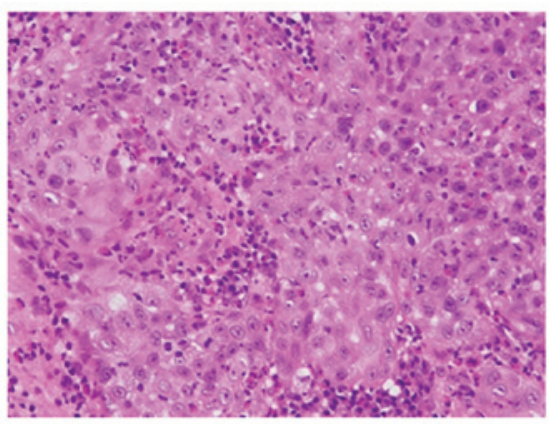

$\mathrm{C}$

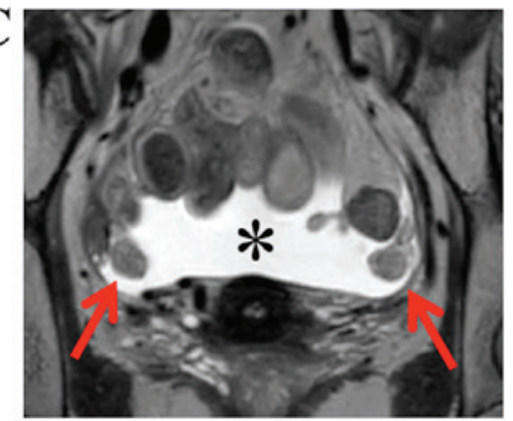

B

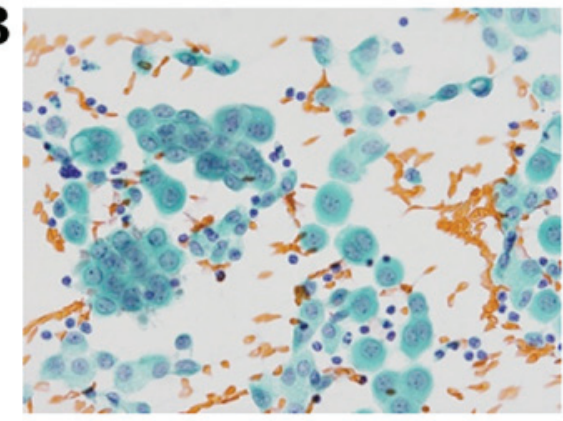

D

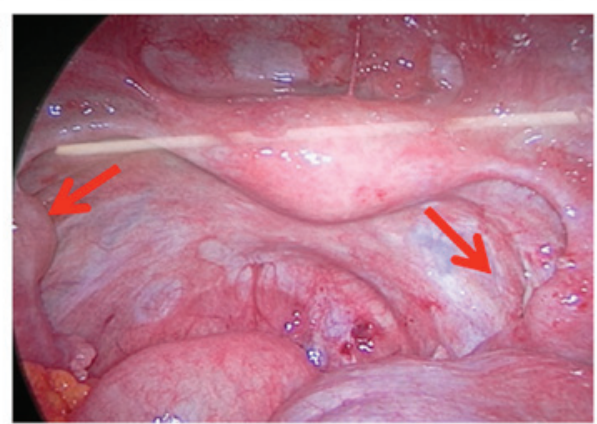

Figure 1. Findings of primary cervical cancer and pre- and intraoperative findings of PMM. (A) Histologic examination of the mass in the uterine cervix indicates squamous cell carcinoma, non-keratinizing type. (B) Cytology after 54 months of CCRT showing atypical binuclear mesothelial cells with irregular nuclei (Papanicolaou staining, x100). (C) T2-weighted MRI in the coronal view showing 20-mm masses in both the salpinges (red arrows), as well as ascites (asterisk). (D) Laparoscopic examination showing bilateral intra-tubal masses (red arrows). PMM, peritoneal malignant mesothelioma; CCRT, concurrent chemoradiotherapy.
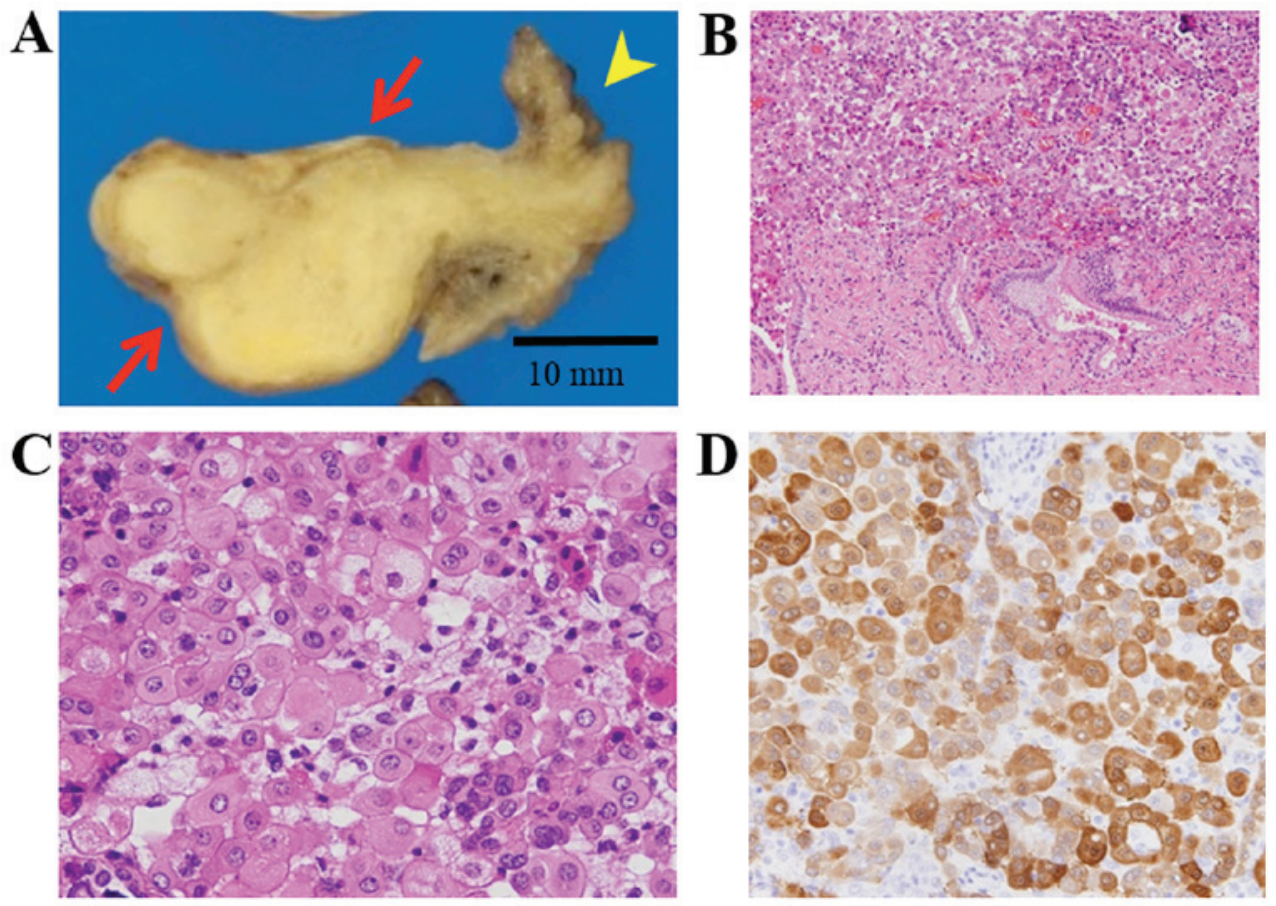

Figure 2. Postoperative findings of PMM. (A) Macroscopic findings show a 20-mm solid mass in the ampulla of the uterine tube (red arrows). The yellow arrowhead shows the fimbriae. (B and C) Histologic examination of the mass in the left salpinx reveals malignant mesothelioma, epithelioid type [H\&E (B) x20 and (C) x60]. (B) There is no continuity between the background tubal epithelium and mesothelioma. (D) IHC of the intra-tubal mass shows positive staining for calretinin. PMM, peritoneal malignant mesothelioma; H\&E, hematoxylin and eosin; IHC, immunohistochemistry.

54 Gray of whole pelvis radiation and 20 Gray/4 fractions of high-dose-rate intracavitary brachytherapy combined with weekly cisplatin administration. After the primary therapy, complete response was achieved and the patient had been followed up constantly with routine medical examinations.
After 54 months of CCRT, transvaginal ultrasonography revealed the existence of ascites at the rectouterine pouch. Two months later, peritoneal cytology by abdominal paracentesis showed mesothelial cells with mild atypia, suggestive of reactive mesothelial cells (Fig. 1B); the patient was diagnosed with 
a reactive mesothelium and was closely followed up. After 62 months of CCRT, MRI indicated 20-mm masses in both of the salpinges, with low intensity on $\mathrm{T} 2$ weighted images, and pelvic ascites (Fig. 1C). The serum levels of SCC and CA125 were $0.9 \mathrm{ng} / \mathrm{ml}$ and $506.1 \mathrm{U} / \mathrm{ml}$, respectively. As the elevation pattern of the serum tumor markers was different from primary cervical SCC and the presence of ascites is rarely seen in recurrence of cervical SCC, we considered the possibility of not recurrence but secondary malignancy. A laparoscopic examination was performed to determine the pathological diagnosis, and it revealed white muddy ascites, bilateral intra-tubal masses (Fig. 1D), and multiple peritoneal dissemination areas of a maximum size of $10 \mathrm{~mm}$. Left salpingectomy and peritoneal biopsy were performed. Intraoperative peritoneal cytology showed atypical mesothelial cells with binuclear and enlarged irregular nuclei. Macroscopically, there was a $20-\mathrm{mm}$ solid mass in the ampulla of the uterine tube (Fig. 2A). Histological examination showed the presence of mesothelial, round tumor cells with mild to moderate pleomorphism and prominent nucleoli, strongly suggesting the possibility of malignant mesothelioma. In the main tumor located intra-tubally, however, there was no continuity between the background tubal epithelium and the malignant mesothelioma (Fig. 2B, C). Immunohistochemistry (IHC) showed positive staining for calretinin (Fig. 2D), thrombomodulin, mesothelin, D2-40, CK20, and glucose transporter 1, and negative staining for $\mathrm{CK} 7$, estrogen receptor, progesterone receptor, pax-8, CD146, CEA, epithelial membrane antigen (EMA), epithelial specific antigen (MOC31), claudin 4, and BRCA1-associated protein-1 (BAP1). Additional examination of fluorescence in situ hybridization (FISH) revealed no findings of homozygous p16 deletion. Based on these findings, the patient was diagnosed with PMM, epithelioid type. She underwent systemic chemotherapy with cisplatin plus pemetrexed, and stable disease status has been obtained for 3 months.

\section{Discussion}

PMM accounts for $17-32 \%$ of mesotheliomas in women, who are usually middle-aged or elderly. The association between the exposure to asbestos and PMM is less strong than it is for pleural mesothelioma, particularly among women (6). Ascites is present in most cases. The present case was observed in a young adult woman who received radiotherapy for cervical cancer and who had no asbestos exposure. Because of the unlikely recurrence pattern from cervical SCC considering both tumor marker levels and the presence of ascites, we performed surgical resection of the tumors. The main location of the malignant mesothelioma is intra-tubal; however, to the best of our knowledge, there are no reports of tubal-origin malignant mesothelioma. We made the diagnosis of PMM because of an absence of continuity between the background tubal epithelium and the tumors, and a presence of multiple peritoneal disseminations. Retrospectively, as the findings of initial peritoneal cytology showed a potential of PMM, we might have been able to suggest the histological examination for the patient earlier. Our experience suggests the importance of histopathological and immunohistochemical examination in cases of an atypical tumorigenesis pattern after primary treatment.
Radiotherapy for cervical cancer seems to increase the risk for developing high-grade endometrial cancer and carcinosarcoma (7). Although radiation therapy for several cancers is known to increase the risk for PMM (8), PMM after radiation therapy for cervical cancer is extremely rare; to the best of our knowledge, only 2 reports have been published so far $(9,10)$. In the previous 2 cases of PMM after radiation therapy for cervical cancer, limited information about pathological findings has been described $(9,10)$. In this report, we present detailed information about the tumor markers, histological subtype, IHC, and p16 homologous deletion status. In particular, tumor markers and IHC data are helpful in the differential diagnosis. The histological subtype, BAP-1 expression on IHC, and p16 homologous deletions on FISH are some of the prognostic factors for PMM $(11,12)$. We believe that our report will contribute to the diagnosis of PMM after radiotherapy for cervical cancer.

In general, the period between the occurrence of primary and secondary malignancies is 10 or more years (3). The mean interval for the development of high-grade endometrial cancer and carcinosarcoma after radiotherapy for cervical cancer was 14 years (7). However, in all the 3 cases, including our current case, the tumor had been diagnosed as PMM within 10 years after radiation therapy for cervical cancer, and our case had the shortest interval, being within 5 years $(9,10)$. We suggest PMM tends to develop within 10 years after radiotherapy in patients with cervical cancer. Physicians need to pay adequate attention for secondary PMM, especially within 10 years after radiotherapy.

In conclusion, we herein report the third case of PMM after radiation therapy for cervical cancer. Our case demonstrates the possibility of PMM occurrence within 10 years after radiotherapy, and indicates the importance of histological and immunohistochemical examination, especially in cases of an atypical tumorigenesis pattern from the primary cancer.

\section{Acknowledgements}

We appreciate the advice and expertise of Dr Kenzo Hiroshoma, Department of Pathology, Tokyo Women's Medical University Yachiyo Medical Center, Dr Toshiaki Kawai, Department of Pathology and Laboratory Medicine, National Defense Medical College, and Dr Teruaki Oka, Division of Pathology, Kanto Central Hospital. We appreciate the advice of Professor Keiichi Fujiwara, Professor Kosei Hasegawa, and Dr Hiroyuki Yoshida, Department of Gynecologic Oncology, Saitama Medical University International Medical Center.

\section{References}

1. Jasani B and Gibbs A: Mesothelioma not associated with asbestos exposure. Arch Pathol Lab Med 136: 262-267, 2012.

2. National Council on Radiation Protection and Measurements (NCRP) Scientific Committee 1-17. Second Primary Cancers and Cardiovascular Disease After Radiotherapy. NCRP Report No. 170. Bethesda, MD, National Council on Radiation Protection and Measurements, 2011.

3. Travis LB, Ng AK, Allan JM, Pui CH, Kennedy AR, Xu XG, Purdy JA, Applegate K, Yahalom J, Constine LS, et al: Second malignant neoplasms and cardiovascular disease following radiotherapy. J Natl Cancer Inst 104: 357-370, 2012.

4. Chaturvedi AK, Engels EA, Gilbert ES, Chen BE, Storm H, Lynch CF, Hall P, Langmark F, Pukkala E, Kaijser M, et al: Second cancers among 104,760 survivors of cervical cancer: Evaluation of long-term risk. J Natl Cancer Inst 99: 1634-1643, 2007. 
5. Kleinerman RA, Boice JD Jr, Storm HH, Sparen P, Andersen A Pukkala E, Lynch CF, Hankey BF and Flannery JT: Second primary cancer after treatment for cervical cancer. An international cancer registries study. Cancer 76: 442-452, 1995.

6. Spirtas R, Heineman EF, Bernstein L, Beebe GW, Keehn RJ Stark A, Harlow BL and Benichou J: Malignant mesothelioma: Attributable risk of asbestos exposure. Occup Environ Med 51: 804-811, 1994.

7. Pothuri B, Ramondetta L, Eifel P, Deavers MT, Wilton A Alektiar K, Barakat R and Soslow RA: Radiation-associated endometrial cancers are prognostically unfavorable tumors: A clinicopathologic comparison with 527 sporadic endometrial cancers. Gynecol Oncol 103: 948-951, 2006.

8. Farioli A, Ottone M, Morganti AG, Compagnone G, Romani F, Cammelli S, Mattioli S and Violante FS: Radiation-induced mesothelioma among long-term solid cancer survivors: A longitudinal analysis of SEER database. Cancer Med 5: 950-959, 2016

9. Babcock TL, Powell DH and Bothwell RS: Radiation-induced peritoneal mesothelioma. J Surg Oncol 8: 369-372, 1976.
10. Beier KM, Gallup DG, Burgess R and Stock RJ: Occurrence of malignant peritoneal mesothelioma after surgery and irradiation for cervical cancer. Gynecol Oncol 17: 375-380, 1984

11. Cerruto CA, Brun EA, Chang D and Sugarbaker PH: Prognostic significance of histomorphologic parameters in diffuse malignant peritoneal mesothelioma. Arch Pathol Lab Med 130: 1654-1661, 2006.

12. Singhi AD, Krasinskas AM, Choudry HA, Bartlett DL, Pingpank JF, Zeh HJ, Luvison A, Fuhrer K, Bahary N, Seethala RR and Dacic S: The prognostic significance of BAP1, NF2, and CDKN2A in malignant peritoneal mesothelioma. Mod Pathol 29: 14-24, 2016. 